\title{
Generation of maximal three-state field-free molecular orientation with terahertz pulses
}

\author{
Hong, Qian Qian; Fan, Li Bao; Shu, Chuan Cun; Henriksen, Niels E.
}

Published in:

Physical Review A

Link to article, DOI:

10.1103/PhysRevA.104.013108

Publication date:

2021

Document Version

Publisher's PDF, also known as Version of record

Link back to DTU Orbit

Citation (APA):

Hong, Q. Q., Fan, L. B., Shu, C. C., \& Henriksen, N. E. (2021). Generation of maximal three-state field-free molecular orientation with terahertz pulses. Physical Review A, 104, [013108].

https://doi.org/10.1103/PhysRevA.104.013108

\section{General rights}

Copyright and moral rights for the publications made accessible in the public portal are retained by the authors and/or other copyright owners and it is a condition of accessing publications that users recognise and abide by the legal requirements associated with these rights.

- Users may download and print one copy of any publication from the public portal for the purpose of private study or research.

- You may not further distribute the material or use it for any profit-making activity or commercial gain

- You may freely distribute the URL identifying the publication in the public portal 


\title{
Generation of maximal three-state field-free molecular orientation with terahertz pulses
}

\author{
Qian-Qian Hong $\odot,{ }^{1}$ Li-Bao Fan, ${ }^{1}$ Chuan-Cun Shu $\odot,{ }^{1,}{ }^{*}$ and Niels E. Henriksen $\odot^{2}$ \\ ${ }^{1}$ Hunan Key Laboratory of Nanophotonics and Devices, \\ Hunan Key Laboratory of Super-Microstructure and Ultrafast Process, \\ School of Physics and Electronics, Central South University, Changsha 410083, China \\ ${ }^{2}$ Department of Chemistry, Technical University of Denmark, Building 207, DK-2800 Kongens Lyngby, Denmark
}

(Received 27 April 2021; revised 22 June 2021; accepted 30 June 2021; published 19 July 2021)

\begin{abstract}
We present a combined analytical and numerical investigation to show how an optimal control field can be designed to generate maximum field-free orientation of molecules for three populated rotational states. Based on a model involving pure rotational ladder-climbing excitation between rotational states, a set of optimal amplitude and phase conditions are analytically derived for the applied control fields. The maximum degree of orientation can be achieved when the field satisfies amplitude and phase conditions at the two transition frequencies. Multiple optimal solutions exist, and to examine these conditions, we devise a quantum coherent control scheme using two terahertz pulses and successfully apply it to the linear polar molecule $\mathrm{HCN}$ at ultracold temperature. The sensitivity of both populations and phases of rotational states to control field parameters, i.e., the detuning, bandwidth, and time delay, is analyzed so as to understand the optimal orientation mechanism. This work thus examines the frequency-domain landscape belonging to optimal pulses.
\end{abstract}

DOI: 10.1103/PhysRevA.104.013108

\section{INTRODUCTION}

Controlling molecular rotations associated with molecular alignment and orientation is an active research area of experimental and theoretical molecular science [1-5], because of its fundamental importance in physics and chemistry. An oriented molecular sample has an asymmetric angular distribution upon reflection, while this distribution for the aligned molecule is symmetric but not isotropic. As a result, achieving molecular orientation since it requires the breakdown of inversion symmetry is more challenging than the well-established alignment in both the adiabatic and nonadiabatic regimes, where the adiabatic limit implies that the duration of the applied laser pulses is much longer than the characteristic timescale of the free rotation of the molecule. Over the years, extensive efforts in theory and experiment have been put into generating a postpulse orientationknown as the field-free orientation of molecules [6-12]. It has potential applications ranging from molecularphase modulators, ultrafast $\mathrm{x}$-ray diffraction, and ultrashort pulse compression to chemical reactivity, nanoscale design, high-order-harmonic generation, and molecular rotational echoes [13-20].

The physical mechanism for generating the field-free orientation is to create a rotational wave packet, which consists of a superposition of rotational states with even and odd angular momentum quantum numbers. Experimentally, alloptical techniques in generating such a field-free orientation have matured thanks to the nonresonant light interactions with molecular polarizability and hyperpolarizability [6-10].

\footnotetext{
*cc.shu@csu.edu.cn, he/him/his
}

As compared with the all-optical techniques, the progress in the realization of the field-free orientation of molecules exclusively using resonant terahertz pulses was relatively slow $[21,22]$ since it was proposed 20 years ago [23-25]. The technical difficulty was previously attributed to using a highly asymmetric half-cycle terahertz pulse, which impulsively transfers angular momentum to the molecule due to a nonzero (time-integrated) area of the short central part [26-31]. Further theoretical investigations have shown that molecules can be oriented through resonant excitation using symmetric terahertz pulses with a zero-time-integrated area. In addition, applying an intense nonresonant ultrashort pulse to align the molecule prior to the resonant terahertz excitation, comprehensive theoretical research has recently resulted in the experimental demonstration of enhanced field-free orientation in the sudden-impact limit [32-35]. However, it remains challenging to obtain a robust field-free orientation without the additional use of an intense nonresonant pulse or a static electric field.

Despite the slow progress in experiments, several theoretical proposals have been directed toward the realization of molecular field-free orientation by exclusively using zeroarea terahertz pulses [36-40]. Furthermore, recent theoretical work has shown that a single half-cycle-zero-area terahertz pulse can result in steady molecular orientation a long time after the pulse is turned off [41]. We recently examined a three-state molecular orientation model with an experimentally available single-cycle terahertz pulse [42]. We found that the theoretical maximum degree of orientation for three populated rotational states can be obtained in the intermediate nonadiabatic limit. The corresponding rotational excitation processes consist of rotational ladder climbing and simultaneous multiphoton absorption. It is a fundamentally 
interesting question whether the pure rotational ladderclimbing excitation can independently generate the maximum orientation while suppressing simultaneous multiphoton absorption processes.

In this paper, we perform a further investigation of the three-state model. Based on an analytical wave function for a three-state system that was used in our previous work [42], we derive amplitude and phase conditions for the control fields, which are required for generating the theoretical maximum degree of orientation for three populated rotational states. We then explain how to use these amplitude and phase conditions and devise a quantum coherent control scheme to examine our theoretical analysis using two terahertz pulses. The proposed coherent control schemes are applied to the linear polar molecule $\mathrm{HCN}$ with four representative simulations. We find that the pure rotational ladder-climbing excitation can induce the maximum degree of orientation as long as the pulses satisfy amplitude and phase conditions at the two transition frequencies. This work provides deep insight into optimal molecular rotations by involving the rotational ladderclimbing excitation mechanism. It also offers an essential reference for designing an experimental scheme toward realizing optimal field-free orientation within a finite rotational Hilbert subspace.

The remainder of this paper is organized as follows. In Sec. II, we describe the theoretical methods for analyzing the three-state-orientation model. We present the results of the numerical simulations and discussion in Sec. III. Finally, we conclude with a brief summary in Sec. IV.

\section{THEORETICAL METHODS}

We consider a general model for producing three populated rotational states by using linearly polarized terahertz pulses $\mathcal{E}(t)$, which turn on at $t_{0}$ and off at $t_{f}$ with $\mathcal{E}\left(t_{0}\right)=\mathcal{E}\left(t_{f}\right)=$ 0 . For the molecule in its electronic and vibrational ground state, the molecular Hamiltonian can be given by $\hat{H}(t)=$ $\hat{H}_{0}+\hat{V}(t)$, where $\hat{H}_{0}=B \hat{J}^{2}$ is the field-free Hamiltonian for a linear molecule with the angular momentum operator $\hat{J}$ and rotational constant $B$ and $\hat{V}(t)$ denotes the interaction Hamiltonian. Within the dipole approximation, the interaction Hamiltonian can be given by $\hat{V}(t)=-\mu \mathcal{E}(t) \cos \theta$, where $\theta$ is the angle between the rotor axis and the pulse polarization. Note that we consider the electric-field strengths of $\mathcal{E}(t)$ in a relatively weak regime. The contribution of the molecular polarizability and hyperpolarizability to the rotational excitation can be ignored.

The time evolution of the system in the interaction picture from the initial time $t_{0}$ to a given time $t$ can be described by a unitary operator $\hat{U}\left(t, t_{0}\right)$, which has a solution $(\hbar=1)$

$$
\hat{U}\left(t, t_{0}\right)=\mathbb{I}-i \int_{t_{0}}^{t} d t^{\prime} \hat{H}_{I}\left(t^{\prime}\right) \hat{U}\left(t^{\prime}, t_{0}\right)
$$

where $\hat{H}_{I}(t)=\exp \left(i \hat{H}_{0} t\right)[-\hat{\mu} \mathcal{E}(t)] \exp \left(-i \hat{H}_{0} t\right)$ and the matrix elements of the dipole operator $\hat{\mu} \operatorname{read} \mu_{J J^{\prime}}=\mu\left\langle J^{\prime} M\right|$ $\cos \theta|J M\rangle \quad$ with $\langle J+1 M|\cos \theta| J M\rangle=\sqrt{(J+1)^{2}-M^{2}}$ $/ \sqrt{(2 J+1)(2 J+3)}$. The time-dependent three-state wave packet after the laser pulse excitation is given by

$$
\left|\psi_{J_{0} M}(t)\right\rangle=\sum_{J^{\prime}=0}^{2} c_{J^{\prime}}\left(t_{f}\right) e^{-i E_{J^{\prime}} M^{t}}\left|J^{\prime} M\right\rangle,
$$

where the rotational eigenstates $\left|J^{\prime}\right\rangle$ satisfy $\hat{H}_{0}\left|J^{\prime}\right\rangle=E_{J^{\prime}}\left|J^{\prime}\right\rangle$ with eigenenergies $E_{J^{\prime}}=B J^{\prime}\left(J^{\prime}+1\right)$ and $c_{J^{\prime}}$ are the expansion coefficients of $\left|J^{\prime}\right\rangle$, which can be obtained by $c_{J^{\prime}}(t)=$ $\left\langle J^{\prime} M\left|\hat{U}\left(t, t_{0}\right)\right| J_{0} M\right\rangle$ with the initial state $\left|J_{0} M\right\rangle$. The degree of orientation after the rotational excitation with the selection rule $\Delta J= \pm 1$ can be written as

$$
\begin{aligned}
\langle\cos \theta\rangle(t)= & 2 \mathcal{M}_{1,0}\left|c_{1}(t)\right|\left|c_{0}(t)\right| \cos \left(\omega_{01} t-\phi_{01}\right) \\
& +2 \mathcal{M}_{2,1}\left|c_{2}(t)\right|\left|c_{1}(t)\right| \cos \left(\omega_{12} t-\phi_{12}\right),
\end{aligned}
$$

where $\mathcal{M}_{J^{\prime}, J}=\left\langle J^{\prime} M|\cos \theta| J M\right\rangle$, the transition frequencies are defined by $\omega_{01}=\left(E_{1}-E_{0}\right)=2 B$ and $\omega_{12}=\left(E_{2}-E_{1}\right)=$ $2 \omega_{01}=4 B$, and the relative phases are $\phi_{01}=\arg \left[c_{1}(t)\right]-$ $\arg \left[c_{0}(t)\right]$ and $\phi_{12}=\arg \left[c_{2}(t)\right]-\arg \left[c_{1}(t)\right]$. This shows that full revivals in the field-free orientation occur at a time interval $\tau=\pi / B$ when $\phi_{12}=2 \phi_{01}+k \pi(k=0, \pm 1, \pm 2, \ldots)$ by generating the coherent superposition of rotational states.

\section{A. The maximum degree of orientation for a three-state model}

Based on the method of Lagrange multipliers, the maximum degree of orientation with the three-state subspace can be estimated by $[43,44]$

$$
\mathcal{L}\left(\left|c_{0}\right|,\left|c_{1}\right|,\left|c_{2}\right|, \lambda\right)=f-\lambda g
$$

where $f=2 \mathcal{M}_{1,0}\left|c_{1} c_{0}\right|+2 \mathcal{M}_{2,1}\left|c_{2} c_{1}\right|$ corresponds to the amplitude of the orientation at the full revivals by considering the relative phases in Eq. (3) to satisfy a relation of $\phi_{12}=2 \phi_{01}+k \pi \quad(k=0, \pm 1, \pm 2, \ldots)$ and $g=\left|c_{0}\right|^{2}+$ $\left|c_{1}\right|^{2}+\left|c_{2}\right|^{2}-1=0$ is a constraint. The extremum of $f$ subject to $g$ can be obtained by satisfying $\nabla \mathcal{L}=0$; then we have

$$
\begin{aligned}
\mathcal{M}_{1,0}\left|c_{1}\right|-\lambda\left|c_{0}\right| & =0, \\
\mathcal{M}_{2,1}\left|c_{2}\right|+\mathcal{M}_{1,0}\left|c_{0}\right|-\lambda\left|c_{1}\right| & =0, \\
\mathcal{M}_{2,1}\left|c_{1}\right|-\lambda\left|c_{2}\right| & =0 .
\end{aligned}
$$

By multiplying each equation in (5) by $\left|c_{0}\right|,\left|c_{1}\right|$, and $\left|c_{2}\right|$, respectively, we have

$$
f-\lambda\left(\left|c_{0}\right|^{2}+\left|c_{1}\right|^{2}+\left|c_{2}\right|^{2}\right)=f-\lambda=0 .
$$

The maximum degree of orientation $f$ corresponds to the maximum value of $\lambda$ governed by Eq. (5). By multiplying the first, second, and third equations in (5) by $\left|c_{0}\right|,-\left|c_{1}\right|$, and $\left|c_{2}\right|$, respectively, we can obtain the relation $\left|c_{0}\right|^{2}+\left|c_{2}\right|^{2}=\left|c_{1}\right|^{2}$ when the maximum degree of orientation $f$ is reached. For the molecules initially in the ground rotational state with $J=$ 0 and $M=0$, we can calculate the matrix elements $\mathcal{M}_{1,0}=$ $\sqrt{1 / 3}$ and $\mathcal{M}_{2,1}=\sqrt{4 / 15}$. As a result, the maximum degree of orientation can be obtained by

$$
\lambda=\frac{\mathcal{M}_{2,1}\left|c_{2}\right|+\mathcal{M}_{1,0}\left|c_{0}\right|}{\left|c_{1}\right|}
$$

which reaches its maximum, 0.7746, with $\left|c_{0}\right|=\sqrt{10} / 6$, $\left|c_{1}\right|=\sqrt{2} / 2$, and $\left|c_{2}\right|=\sqrt{2} / 3$. Figure 1 illustrates the principle of field-free time-dependent orientation. By generating a coherent superposition of three populated rotational states 


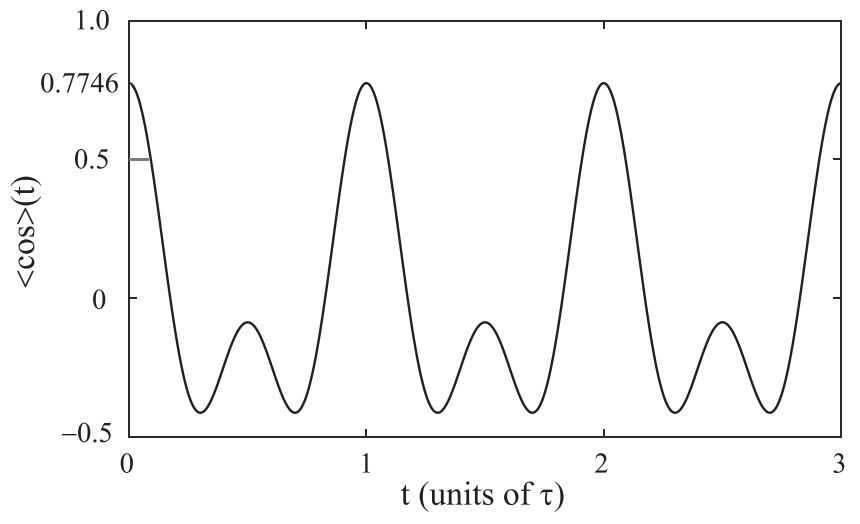

FIG. 1. The principle of generating maximal three-state fieldfree molecular orientation. A coherent superposition of three populated rotational states with $p_{0}=\left|c_{0}\right|^{2}=0.278, p_{1}=\left|c_{1}\right|^{2}=$ 0.5 , and $p_{2}=\left|c_{2}\right|^{2}=0.222$ is created at $t=0$, leading to full revivals with the maximum of 0.7746 at a time interval $\tau$.

with $p_{0}=\left|c_{0}\right|^{2}=0.278, p_{1}=\left|c_{1}\right|^{2}=0.5$, and $p_{2}=\left|c_{2}\right|^{2}=$ 0.222 at $t=0$ while satisfying the phase relation between states, the maximum degree of orientation with the value of 0.7746 can be generated as defined in Eq. (3), showing full revivals of the field-free orientation at a time interval $\tau$.

\section{B. An analytical solution for the rotational wave packet}

We now recall the theoretical analysis used in our previous work [42] to show the dependence of $c_{J^{\prime}}(t)$ on the control field $\mathcal{E}(t)$. We write the Hamiltonian in the interaction picture without using the rotating wave approximation [45-47]

$\hat{H}_{I}(t)=-\left(\begin{array}{ccc}0 & \mu_{10} \mathcal{E}(t) e^{-i \omega_{01} t} & 0 \\ \mu_{10} \mathcal{E}(t) e^{i \omega_{01} t} & 0 & \mu_{21} \mathcal{E}(t) e^{-i \omega_{12} t} \\ 0 & \mu_{21} \mathcal{E}(t) e^{i \omega_{12} t} & 0\end{array}\right)$

We expand the unitary operator $\hat{U}\left(t, t_{0}\right)$ by using the Magnus expansion [48]

$$
\hat{U}\left(t, t_{0}\right)=\exp \left[\sum_{n=1}^{\infty} \hat{S}^{(n)}(t)\right]
$$

where the first leading term is given by

$$
\hat{S}^{(1)}(t)=-i \int_{t_{0}}^{t} d t_{1} \hat{H}_{I}\left(t_{1}\right)
$$

The corresponding time-dependent wave function $\left|\psi^{(1)}(t)\right\rangle \equiv$ $\sum_{J^{\prime}=0}^{2} c_{J^{\prime}}^{(1)}\left|J^{\prime} 0\right\rangle=\hat{U}^{(1)}\left(t, t_{0}\right)|00\rangle$ starting from the ground rotational state $|00\rangle$ can be given by [42]

$$
\begin{aligned}
\left|\psi^{(1)}(t)\right\rangle= & \frac{\left[\left|\theta_{2}(t)\right|^{2}+\left|\theta_{1}(t)\right|^{2} \cos \theta_{12}(t)\right]}{\theta_{12}^{2}(t)}|00\rangle \\
& +\frac{i \theta_{1}(t) \sin \theta_{12}(t)}{\theta_{12}(t)}|10\rangle \\
& +\frac{\theta_{1}(t) \theta_{2}(t)}{\theta_{12}^{2}(t)}\left[\cos \theta_{12}(t)-1\right]|20\rangle
\end{aligned}
$$

where $\theta_{12}(t)=\sqrt{\left|\theta_{1}(t)\right|^{2}+\left|\theta_{2}(t)\right|^{2}}$ with

$$
\theta_{1}(t)=\mu_{10} \int_{t_{0}}^{t} d t^{\prime} \mathcal{E}\left(t^{\prime}\right) e^{i \omega_{01} t^{\prime}}
$$

and

$$
\theta_{2}(t)=\mu_{21} \int_{t_{0}}^{t} d t^{\prime} \mathcal{E}\left(t^{\prime}\right) e^{i \omega_{12} t^{\prime}}
$$

Thus $\theta_{i}(t)$ is the transition dipole moment times the Fourier transform of the electric field at the transition frequency. A rotational ladder-climbing mechanism is involved in generating the superposition of rotational states in Eq. (11) [49]. That is, the molecule is excited resonantly by a one-photon transition from $|00\rangle$ to $|10\rangle$ followed by a one-photon transition from $|10\rangle$ to $|20\rangle$.

\section{The amplitude and phase conditions}

Based on the above analysis, the maximum degree of orientation in Eq. (7) requires that $\theta_{1}\left(t_{f}\right)$ and $\theta_{2}\left(t_{f}\right)$ satisfy the following relations:

$$
\begin{aligned}
\left|c_{0}^{(1)}\left(t_{f}\right)\right| & \equiv\left|\frac{\left|\theta_{2}\left(t_{f}\right)\right|^{2}+\left|\theta_{1}\left(t_{f}\right)\right|^{2} \cos \theta_{12}\left(t_{f}\right)}{\theta_{12}^{2}\left(t_{f}\right)}\right|=\frac{\sqrt{10}}{6} \\
\left|c_{1}^{(1)}\left(t_{f}\right)\right| & \equiv\left|\frac{i \theta_{1}\left(t_{f}\right) \sin \theta_{12}\left(t_{f}\right)}{\theta_{12}\left(t_{f}\right)}\right|=\frac{\sqrt{2}}{2} \\
\left|c_{2}^{(1)}\left(t_{f}\right)\right| & \equiv\left|\frac{\theta_{1}\left(t_{f}\right) \theta_{2}\left(t_{f}\right)}{\theta_{12}^{2}\left(t_{f}\right)}\left[\cos \theta_{12}\left(t_{f}\right)-1\right]\right|=\frac{\sqrt{2}}{3}
\end{aligned}
$$

From Eq. (14), we can derive

$$
\sum_{\ell=0}^{4} a_{\ell}\left[\frac{\left|\theta_{2}\left(t_{f}\right)\right|}{\left|\theta_{1}\left(t_{f}\right)\right|}\right]^{\ell}=0
$$

with the coefficients $a_{0}=2 / 9, a_{1}=a_{3}=-2 \sqrt{2} / 3, a_{2}=$ $17 / 18$, and $a_{4}=13 / 18$.

By setting $s=\left|\theta_{2}\left(t_{f}\right)\right| /\left|\theta_{1}\left(t_{f}\right)\right|$, there exist two real solutions to Eq. (15) with $s_{1}=0.9967$ and $s_{2}=0.3087$. To that end, we obtain two conditions for generating the maximum orientation, i.e.,

$$
\begin{aligned}
& \left|\theta_{1}\left(t_{f}\right)\right|=\frac{\left|\arccos \left(1-\frac{\sqrt{2}}{3 s_{1}}-\frac{\sqrt{2} s_{1}}{3}\right)+2 j \pi\right|}{\sqrt{1+s_{1}^{2}}}, \\
& \left|\theta_{2}\left(t_{f}\right)\right|=s_{1}\left|\theta_{1}\left(t_{f}\right)\right|,
\end{aligned}
$$

and

$$
\begin{aligned}
\left|\theta_{1}\left(t_{f}\right)\right| & =\frac{\left|\arccos \left(1-\frac{\sqrt{2}}{3 s_{2}}-\frac{\sqrt{2} s_{2}}{3}\right)+2 j \pi\right|}{\sqrt{1+s_{2}^{2}}}, \\
\left|\theta_{2} t_{f}\right| & =s_{2}\left|\theta_{1}\left(t_{f}\right)\right|,
\end{aligned}
$$

where $j=0, \pm 1, \pm 2, \ldots$. To make the relative phases in Eq. (3) meet the relation $\phi_{12}=2 \phi_{01}+k \pi(k=$ $0, \pm 1, \pm 2, \ldots), \theta_{1}\left(t_{f}\right)$ and $\theta_{2}\left(t_{f}\right)$ in Eq. (11) are required to satisfy the phase condition

$$
2 \arg \left[\theta_{1}\left(t_{f}\right)\right]-\arg \left[\theta_{2}\left(t_{f}\right)\right]=\left(k \pm \frac{1}{2}\right) \pi .
$$


Note that the theoretical maximum value for the degree of orientation is independent of the excitation scheme. The above amplitude and phase conditions only work to generate the rotational wave packet subject to the first-order Magnus description in Eq. (11). As can be seen from Eqs. (16) and (17), there exist multiple optimal combinations of the amplitude and phase of the control fields, capable of generating the maximal three-state field-free molecular orientation. There is no need for any prior knowledge in choosing one or the other of the analytical conditions, as both of them lead to the same populations of three rotational states. The physical picture behind the two solutions can be analyzed with Eq. (14). The large value of $\theta_{1}\left(t_{f}\right)$ with the second condition corresponds to a strong Rabi coupling between states $J=0$ and 1, indicating that Rabi oscillations occur while resulting in the same population distributions among states after the pulse turns off.

\section{A coherent control scheme with the use of two terahertz pulses}

To use the above amplitude and phase conditions, we design a quantum coherent control scheme by using two terahertz pulses. From the frequency-domain point of view, the complex values of $\theta_{1}\left(t_{f}\right)$ and $\theta_{2}\left(t_{f}\right)$ are related to the Fourier transform of the control field $\mathcal{E}(t)$,

$$
E(\omega) \equiv A(\omega) e^{i \phi(\omega)}=\int_{t_{0}}^{t_{f}} d t^{\prime} \mathcal{E}\left(t^{\prime}\right) e^{-i \omega t^{\prime}}
$$

where $A(\omega)$ and $\phi(\omega)$ denote the spectral amplitude and spectral phase of the field, respectively. By comparing Eq. (19) with Eqs. (12) and (13), we can obtain the two relations

$$
\theta_{1}^{*}\left(t_{f}\right)=\mu_{10} A\left(\omega_{01}\right) e^{i \phi\left(\omega_{01}\right)}
$$

and

$$
\theta_{2}^{*}\left(t_{f}\right)=\mu_{21} A\left(\omega_{12}\right) e^{i \phi\left(\omega_{12}\right)} .
$$

Thus the amplitude and phase conditions can be satisfied by optimizing the values of $A\left(\omega_{01}\right), A\left(\omega_{12}\right), \phi\left(\omega_{01}\right)$, and $\phi\left(\omega_{12}\right)$, and all pulses that satisfy Eqs. (16)-(18) will give the maximum degree of orientation within the three-state model.

To be specific, we use two Gaussian terahertz pulses to design the complex spectral field by

$$
E(\omega)=\sum_{i=1,2} A_{i} e^{-\frac{\left(\omega-\omega_{i}\right)^{2}}{2 \Delta \omega_{i}^{2}}} e^{i \phi_{i}(\omega)} e^{-i \omega \tau_{i}},
$$

where $A_{i}, \omega_{i}, \Delta \omega_{i}, \phi_{i}$, and $\tau_{i}$ denote the amplitude, fundamental frequency, bandwidth, spectral phase, and center time of the $i$ th pulse, respectively. By setting the values of $\omega_{1}$ and $\omega_{2}$ to the transition frequencies $\omega_{01}$ and $\omega_{12}$, the optimal amplitudes of $\theta_{1}\left(t_{f}\right)$ and $\theta_{2}\left(t_{f}\right)$ can be obtained by scaling the amplitudes $A_{1}$ and $A_{2}$, and the phase conditions can be satisfied by modulating the combined values of $\phi_{1}\left(\omega_{01}\right)-\omega_{01} \tau_{1}$ and $\phi_{2}\left(\omega_{12}\right)-\omega_{12} \tau_{2}$. Note that the maximum orientation is only obtained when the two pulses do not overlap in frequency space, and therefore two narrow-bandwidth terahertz pulses consisting of multiple cycles will be required to satisfy the criteria of Eqs. (16) and (17). This implies that the present two-pulse scheme is different from our previous work [42] using a single-cycle terahertz pulse, for which the rotational

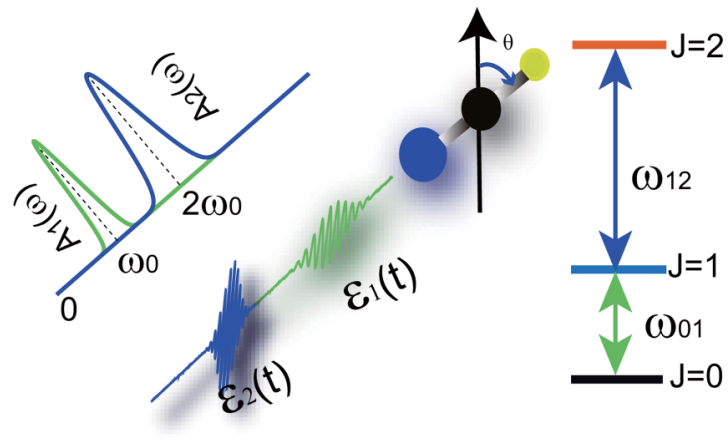

FIG. 2. Schematic of rotational excitation of a linear polar molecule within a three-state model by using two terahertz pulses. The first pulse $\mathcal{E}_{1}(t)$ is centered at $t=0$ with a fundamental frequency $\omega_{0}$, and the second pulse has a time delay $\tau_{0}$ and a center frequency of $2 \omega_{0} . A_{1}(\omega)$ and $A_{2}(\omega)$ correspond to the spectral amplitudes of the pulses. $\theta$ denotes the angle between the rotor axis and the polarization of the pulsed fields. The three-state model consists of states with rotational quantum numbers of $J=0,1$, and 2. $\omega_{01}$ and $\omega_{12}$ are the transition frequencies between states.

excitation involves optical processes governed by high-order Magnus terms, going beyond the present rotational ladderclimbing mechanism.

\section{RESULTS AND DISCUSSION}

We now perform simulations to examine the above model by using two terahertz pulses, as illustrated in Fig. 2, which are applied to the linear polar molecule $\mathrm{HCN}$. The pulses used are composed of the fundamental frequency $\omega_{1}=\omega_{0}$ and the second-harmonic frequency $\omega_{2}=2 \omega_{0}$. We consider two pulses with the same bandwidth $\Delta \omega_{1}=\Delta \omega_{2}=\Delta \omega$. The optimal amplitudes of $A_{1}$ and $A_{2}$ are chosen with $A_{1}=$ $\left|\theta_{1}\left(t_{f}\right)\right| / \mu_{10}$ and $A_{2}=\left|\theta_{2}\left(t_{f}\right)\right| / \mu_{21}$. We fix the center time of the first pulse at $\tau_{1}=0$ and set the second one at $\tau_{2}=\tau_{0}$. Thus the time-dependent electric field $\mathcal{E}(t)$ can be given by

$$
\begin{aligned}
\mathcal{E}(t)= & \frac{1}{\pi} \operatorname{Re}\left\{\int _ { 0 } ^ { \infty } d \omega \left[\frac{\left|\theta_{1}\left(t_{f}\right)\right|}{\mu_{10}} e^{-\frac{\left(\omega-\omega_{0}\right)^{2}}{2 \Delta \omega^{2}}} e^{i \phi_{1}}\right.\right. \\
& \left.\left.+\frac{\left|\theta_{2}\left(t_{f}\right)\right|}{\mu_{21}} e^{-\frac{\left(\omega-2 \omega_{0}\right)^{2}}{2 \Delta \omega^{2}}} e^{i \phi_{2}} e^{-i \omega \tau_{0}}\right] e^{i \omega t}\right\} .
\end{aligned}
$$

Based on the above analysis, we find that the spectral amplitude and phase at the two transition frequencies is all that matters. The frequency-domain shaping analysis from Eq. (19) to Eq. (23) helps us understand how to design the control fields to satisfy the amplitude and phase conditions. To that end, we can give the time-dependent control field in Eq. (23) by

$$
\begin{aligned}
\mathcal{E}(t)= & \sqrt{\frac{2}{\pi}} \frac{1}{\tau}\left\{\frac{\left|\theta_{1}\left(t_{f}\right)\right|}{\mu_{10}} e^{-\frac{t^{2}}{2 \tau^{2}}} \cos \left(\omega_{0} t+\phi_{1}\right)\right. \\
& \left.+\frac{\left|\theta_{2}\left(t_{f}\right)\right|}{\mu_{21}} e^{-\frac{\left(t-\tau_{0}\right)^{2}}{2 \tau^{2}}} \cos \left[2 \omega_{0}\left(t-\tau_{0}\right)+\phi_{2}\right]\right\},
\end{aligned}
$$

where the pulse duration is defined by $\tau=1 / \Delta \omega$. Thus the control scheme shown in Fig. 2 corresponds to the commonly used pump-pump control in experiments involving two time-delayed pulses composed of the fundamental frequency 

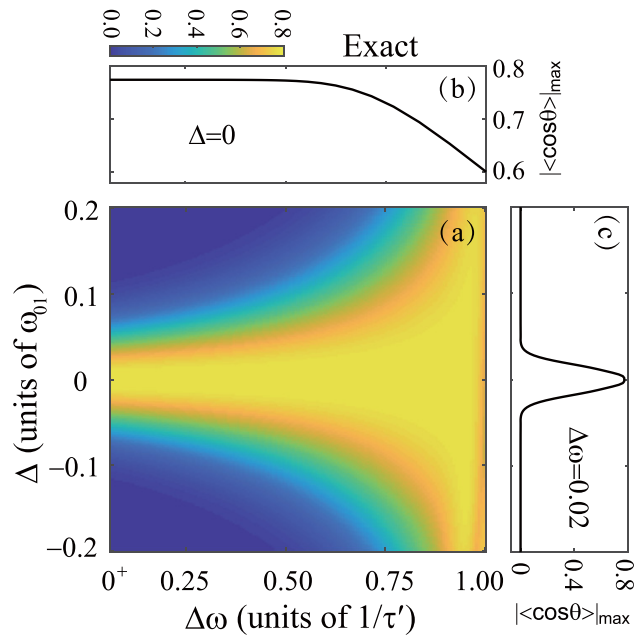
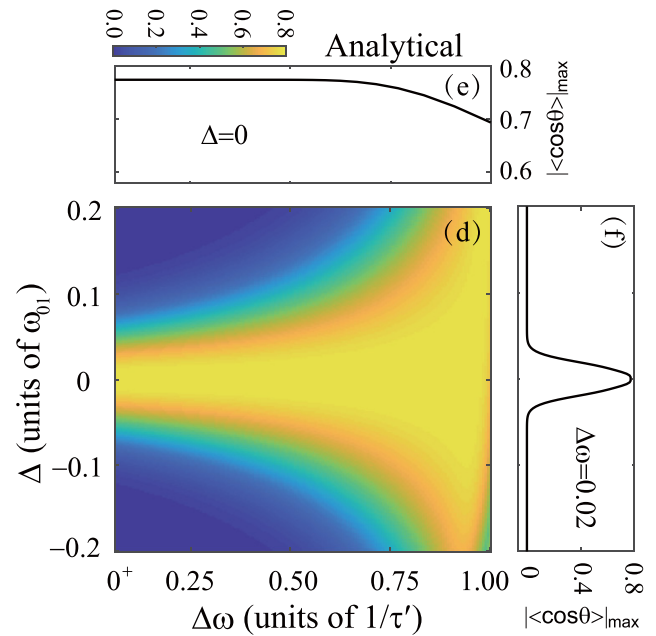

FIG. 3. Numerical simulations with two zero-delayed pulses using the condition in Eq. (16). The maximum orientation values $|\langle\cos \theta\rangle|_{\max }$ are shown vs the bandwidth and the detuning of the pulses. The left panels [(a)-(c)] correspond to the exact simulations using the timedependent unitary operator defined by Eq. (1). The right panels [(d)-(f)] correspond to the analytical simulations using Eq. (11). $1 / \tau^{\prime}=2 \omega_{01} / \pi$ used as the unit corresponds to the bandwidth of a single-cycle pulse, i.e., the shortest possible pulse of a given wavelength.

and the second-harmonic frequency. The amplitude and phase conditions in Eqs. (16)-(18) can be fulfilled by controlling the values of the amplitude and center frequency of the pulses while fixing the phase. That is, the center frequency, strength, and absolute phase of the laser fields can be used as the control parameters to generate the maximum degree of orientation.

\section{A. Simulations with two zero-delayed pulses}

We first examine the case of the time delay $\tau_{0}=0$, i.e., the two pulses act on the molecule simultaneously. The first amplitude condition [Eq. (16)] is used by considering $j=0$, i.e., $\left|\theta_{1}\left(t_{f}\right)\right|=0.3412 \pi$ and $\left|\theta_{2}\left(t_{f}\right)\right|=0.3401 \pi$. The phases are fixed at $\phi_{1}=\phi_{2}=-\pi / 2$ by Eq. (18). Figure 3 shows the dependence of the maximal orientation on the bandwidth $\Delta \omega$ and the detuning $\Delta=\omega_{0}-\omega_{01}$, in which the exactly calculated results using Eq. (1) are compared with the analytical results by using the first-order Magnus approximation. Both simulations show the maximum value of the orientation and its dependence on the parameters, and the exact result and the analytical result match better and better as the bandwidth becomes narrower. To further show this change, Figs. 3(b) and 3(e) show the maximum degree of orientation versus the bandwidth by fixing $\Delta=0$, and Figs. 3(c) and 3(f) exhibit its dependence on detuning by fixing the bandwidth at $\Delta \omega=$ $0.02 / \tau^{\prime}$ with $\tau^{\prime}=\pi / 2 \omega_{01}$. In the broad-bandwidth regime, the maximum orientation value is smaller than the theoretical maximum 0.7746 , and there are some slight differences between the exact and analytical results. The former can be attributed to the overlap between the frequency distributions of the two pulses. That is, the fundamental-frequency pulse with a broad bandwidth also results in transitions between the states $|10\rangle$ and $|20\rangle$, and the second-harmonic one for the same reason also induces transitions between the states $|00\rangle$ and $|10\rangle$. The latter is caused by optical transitions beyond the rotational ladder climbing, as discussed in our previous work [42]. The simultaneous two-photon transitions from $|00\rangle$ to $|20\rangle$ play a role.
To gain insight into the underlying excitation mechanism, Fig. 4 shows the final populations and phases versus the bandwidth by setting the detuning $\Delta=0$, which corresponds to the orientation in Figs. 3(b) and 3(e). The populations and phases of the rotational states are strongly dependent on the
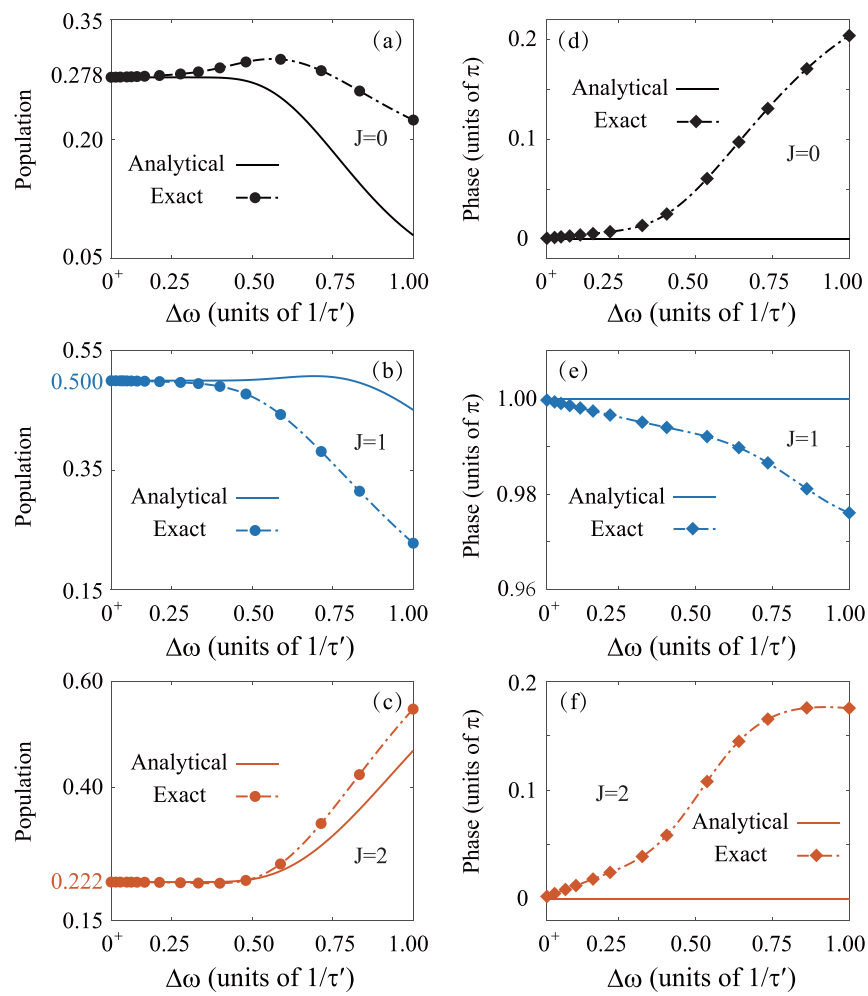

FIG. 4. The final (a)-(c) populations and (d)-(f) phases of the three rotational states $|00\rangle,|10\rangle$, and $|20\rangle$ vs the bandwidths of the pulses at a detuning $\Delta=0$. The exact (dash-dotted lines) populations and phases, i.e., $\left|c_{J^{\prime}}\left(t_{f}\right)\right|^{2}$ and $\arg \left[c_{J^{\prime}}\left(t_{f}\right)\right]$, are compared with the analytical (solid lines) ones, i.e., $\left|c_{J^{\prime}}^{(1)}\left(t_{f}\right)\right|^{2}$ and $\arg \left[c_{J^{\prime}}^{(1)}\left(t_{f}\right)\right]$. 

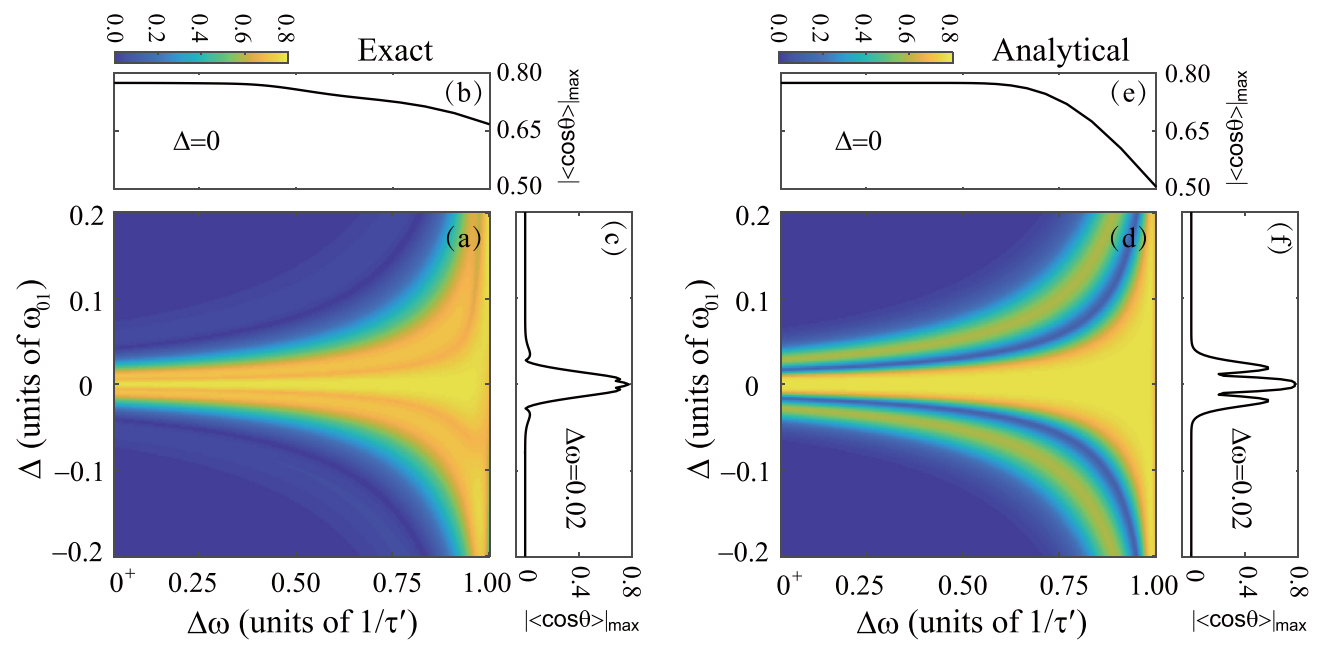

FIG. 5. (a)-(f) The same simulations as in Fig. 3, but for the second condition [Eq. (17)].

pulse bandwidths and gradually converge to the maximum theoretical values by decreasing the pulse bandwidths, leading to the theoretical maximum degree of orientation. We can see that there are visible differences between the exact results and analytical results by using the first-order approximation, indicating that the optical transitions via the higher-order terms in the Magnus expansion occur in the broad-bandwidth regime. However, when the laser pulses turn into the narrowbandwidth regime, the exact results are in good agreement with the analytical model, indicating that the optical processes via high-order Magnus terms are suppressed. As a result, the optical transitions via the rotational ladder climbing described by Eq. (11) determine the orientation dynamics in the narrowbandwidth regime.

Figures 5 and 6 show the same simulations as in Fig. 3 and 4 , now for the second optimal condition [Eq. (17)] with $j=0$, i.e., $\left|\theta_{1}\left(t_{f}\right)\right|=0.7021 \pi$ and $\left|\theta_{2}\left(t_{f}\right)\right|=0.2167 \pi$. The results in Figs. 5 and 6 show similar behaviors to those in Figs. 3 and 4, but there is a visible difference between the exact simulations and the first-order analytical ones. The maximum orientation for both simulations is highly sensitive to both detuning and bandwidth. We first examine the case of $\Delta=0$, for which the exact results can reach the theoretical maximum degree of orientation, which is the same as the analytical one in the narrow-bandwidth regime; see details in Figs. 5(b) and 5(e) and in Fig. 6. By comparing the phases of the second condition in Figs. 6(d)-6(f) with the first one in Figs. 4(d)-4(f), it is interesting to see that the phase of the ground rotational state flips by $\pi$ after the pulse radiations. This phenomenon can be explained by using Eq. (11) with $\theta_{1}\left(t_{f}\right)=0.7021 \pi$ and $\theta_{2}\left(t_{f}\right)=0.2167 \pi$, which does lead to a phase flip for the ground state $|00\rangle$, showing an essential difference from that satisfying the first amplitude condition. In the broad-bandwidth regime, the differences in both populations and phases become obvious because of the pulse overlap in the frequency domain and the optical transitions via high-order terms in the Magnus expansion, which reduce the orientation values below the theoretical maximum.

As shown in Fig. 5, the analytically calculated value of the orientation shows strong oscillations as a function of de- tuning even in the narrow-bandwidth regime. However, this phenomenon is not clearly visible in the exact simulations. Figure 7 shows the population and phase dependence on the detuning at $\Delta \omega=0.02 / \tau^{\prime}$, corresponding to the orientations in Figs. 5(c) and 5(f). We can see that the exactly calculated population-against-detuning changes for the state $|20\rangle$ follow those of the analytical one well. The visible difference in phases implies that the optical transition processes via highorder Magnus terms play a role even in the narrow-bandwidth region, as shown in Fig. 7(f). For the state $|10\rangle$, the analytically calculated population shows strong Rabi oscillations
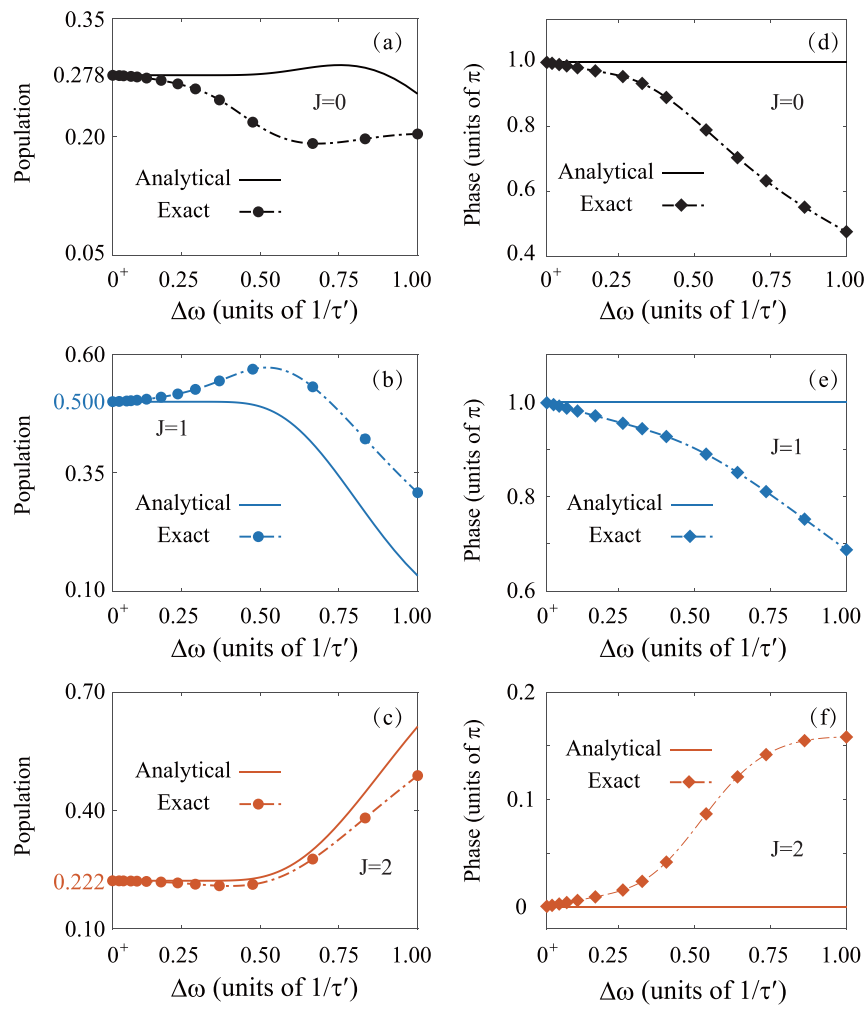

FIG. 6. (a)-(f) The same simulations as in Fig. 4, but for the second condition [Eq. (17)]. 

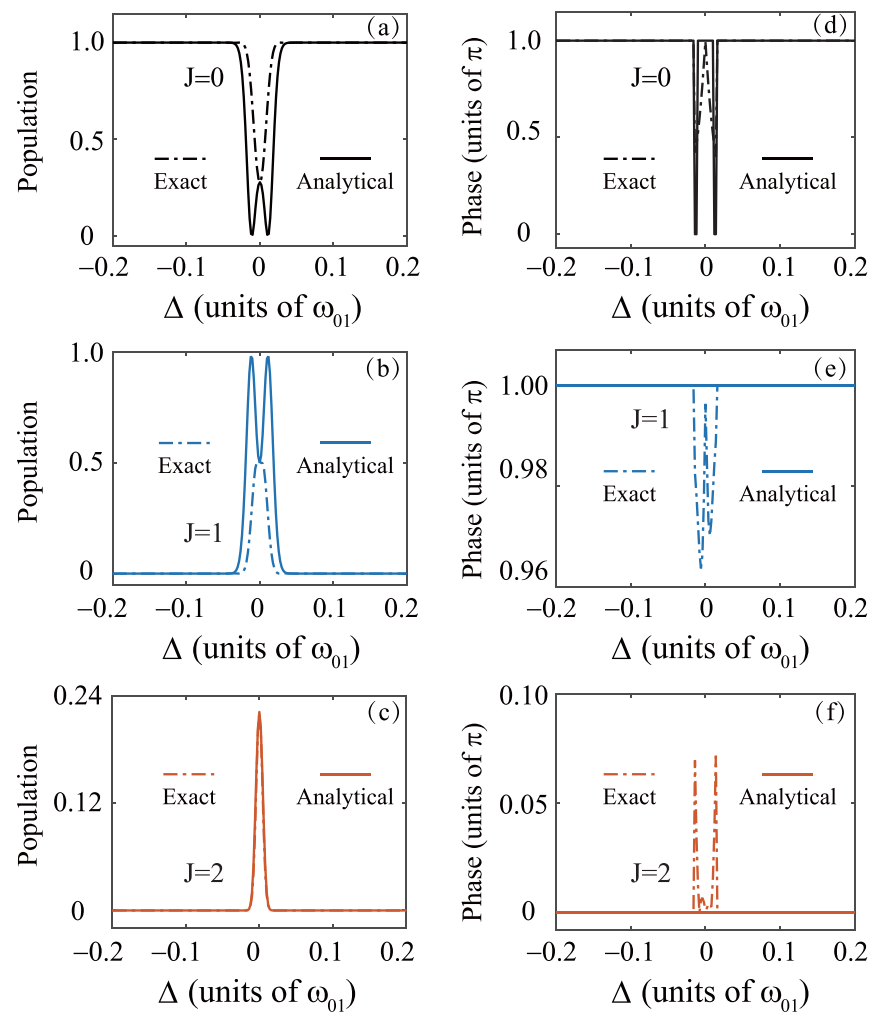

FIG. 7. The dependence of (a)-(c) populations and (d)-(f) phases on the detuning for the control field with a narrow bandwidth of $\Delta \omega=0.02 / \tau^{\prime}$. The dashed lines show the exactly calculated populations and phases, i.e., $\left|c_{J^{\prime}}\left(t_{f}\right)\right|^{2}$ and $\arg \left[c_{J^{\prime}}\left(t_{f}\right)\right]$, and the solid lines correspond to analytical ones, $\left|c_{J^{\prime}}^{(1)}\left(t_{f}\right)\right|^{2}$ and $\arg \left[c_{J^{\prime}}^{(1)}\left(t_{f}\right)\right]$. Note that the phases for larger detunings where the populations of the states $|10\rangle$ and $|20\rangle$ are nearly zero are meaningless and therefore are set to zero in our simulations.

concerning the detuning, which is caused by a larger Rabi coupling between the states $|00\rangle$ and $|10\rangle$ in the second case with $\theta_{1}\left(t_{f}\right)=0.7021 \pi$ than that in the first case with $\theta_{1}\left(t_{f}\right)=$
$0.3412 \pi$. The analytically calculated phases as expected from Eq. (11) in Figs. 7(d)-7(f) are unchanged for small changes in detuning. The corresponding optical transition processes via higher-order Magnus terms strongly affect the phases of rotational states. As a result, the oscillations of the analytically calculated orientations observed in Fig. 5(f) are suppressed by optical processes beyond the first-order Magnus description. For the excitation with extremely small detuning, i.e., $\Delta \approx 0$, the exactly calculated results nicely fit the analytical predictions by satisfying the amplitude and phase conditions of the control fields.

\section{B. Simulations with two time-delayed pulses}

We further examine the scheme by considering two timedelayed terahertz pulses. To satisfy both amplitude and phase conditions, we perform the following simulations using two pulses with a narrow bandwidth of $\Delta \omega=0.02 / \tau^{\prime}$ with $\tau^{\prime}=$ $\pi / 2 \omega_{01}$. We apply the first condition [Eq. (16)] to the simulations and fix the phases $\phi_{1}=\phi_{2}=0$. Figure 8 shows the maximum value of the orientation versus the delay time $\tau_{0}$ and detuning $\Delta$ of the pulses. We can see that the maximum orientation values are highly sensitive to the two laser parameters. At the excitation condition of $\Delta=0$, the orientation values can reach the theoretical maximum at $\tau_{0}=0.5 \tau^{\prime}$ and $1.5 \tau^{\prime}$, where the phases $\phi_{1}, \phi_{2}$, and $\omega_{12} \tau_{0}$ satisfy the phase condition in Eq. (18), and the exactly calculated results are in good agreement with the analytical ones. To see how the delay time affects the orientation values, Fig. 9 shows the populations and phases of the three rotational states versus the time delay after the pulses are turned off. We can see that the populations stay unchanged as the time delay varies, and the phase of the state $|20\rangle$ changes as expected from Eqs. (11) and (23). Note that the exactly calculated results in Figs. 8(a)-8(c) follow the analytical ones in Figs. 8(d)-8(f) very well, only with slight differences concerning detuning.

Figures 10 and 11 display the same simulations as Figs. 8 and 9 by satisfying the second condition [Eq. (17)]. In both
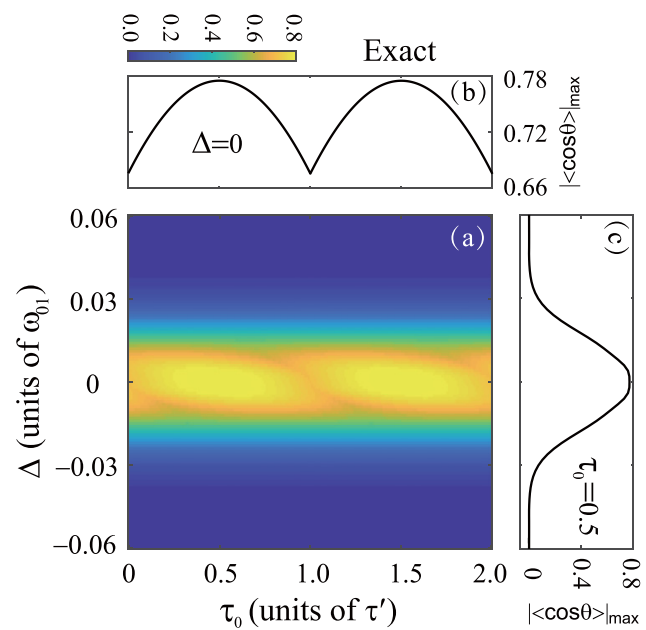
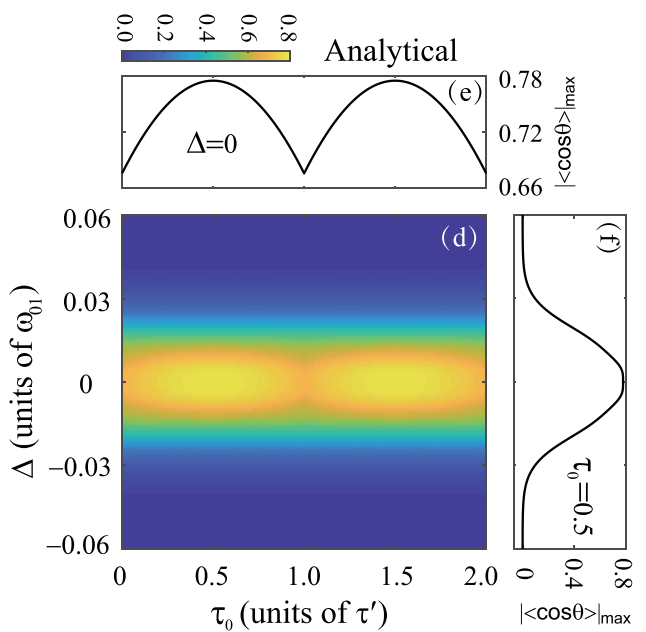

FIG. 8. Numerical simulations with two time-delayed pulses for the first condition [Eq. (16)] with a narrow bandwidth $\Delta \omega=0.02 / \tau^{\prime}$. The maximum orientation values $|\langle\cos \theta\rangle|_{\max }$ are shown vs the time delay and the detuning of the pulses. The left panels [(a)-(c)] correspond to the exact simulations using the time-dependent unitary operator in Eq. (1). The right panels [(d)-(f)] correspond to the analytical simulations using Eq. (11). 

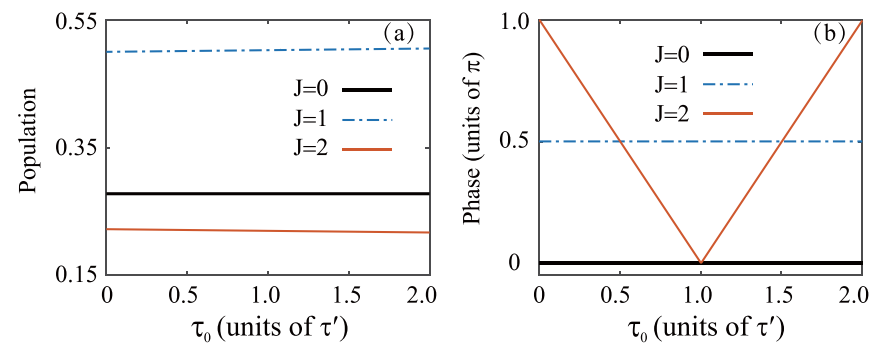

FIG. 9. The dependence of exactly calculated (a) populations and (b) phases, i.e., $\left|c_{J^{\prime}}\left(t_{f}\right)\right|^{2}$ and $\arg \left[c_{J^{\prime}}\left(t_{f}\right)\right]$, on the time delay $\tau_{0}$ for $\Delta=0$, corresponding to the orientation in Fig. 8(b).

simulations, the maximum values of orientation exhibit strong dependences on the laser parameters, i.e., detuning and time delay. For the resonant excitation with $\Delta=0$, the exactly calculated orientation in Fig. 10(b) is consistent with the analytical result in Fig. 10(e). The corresponding populations and phases in Fig. 11 show the same behaviors as those in Fig. 9, i.e., changing the delay time leads to a phase change of the state $|20\rangle$. When we look at the orientation and its dependence on the detuning for a given time delay, the exact results are different from the analytical ones. The latter show clear oscillations concerning the detuning in Fig. 10(f), whereas this phenomenon is strongly suppressed in Fig. 10(c). As analyzed for the second case of the zero delay in Fig. 7, the underlying physics can be attributed to the strong laser coupling between the states $|00\rangle$ and $|20\rangle$, where the high-order Magnus terms play roles for both populations and phases. As a result, the second amplitude and phase conditions of Eqs. (17) and (18) can only be used for extremely small detuning.

Note that in the above simulations, two kinds of conditions in Eqs. (16)-(18) are examined for the case of $j=0$, i.e., $\theta_{1}\left(t_{f}\right)$ and $\theta_{2}\left(t_{f}\right)$, within the range of $[0, \pi]$. In principle, the theoretical conditions of Eqs. (16)-(18) can be analyzed for the case of $j>0$, for which the Rabi couplings between states [i.e., the off-diagonal elements in Eq. (8)] become stronger than those for $j=0$. As a result, the effect of optical excitations by high-order Magnus terms on the orientation may become more pronounced. Therefore the requirements
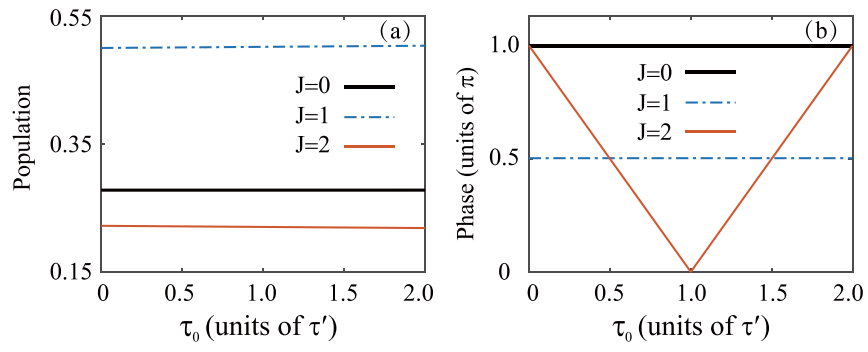

FIG. 11. (a) and (b) The same simulations as in Fig. 9, but for the second condition [Eq. (17)].

that lead to the maximum degree of orientation are strictly limited for using the two conditions. To that end, the experimental realization would be more accessible when $j=0$ in Eqs. (16)-(18).

\section{CONCLUSION}

We performed a combined analytical and numerical analysis to find optimal control fields for achieving the maximum field-free molecular orientation within a three-state model. Using the first-order Magnus expansion to the time-dependent unitary operator, we obtained an analytical solution for a three-state time-dependent wave function. We then derived amplitude and phase conditions, i.e., Eqs. (16)-(18), for the control fields, resulting in the theoretical maximum orientation value with an optimal combination of populations and phases for the three rotational states. Based on the amplitude and phase conditions, we suggested a quantum coherent control scheme and successfully applied it to the linear polar molecules HCN with four different simulations. Multiple optimal solutions exist, and we investigated the frequency-domain landscape of the optimal terahertz pulses. As a result, we showed how pure rotational ladder-climbing excitation could generate the maximal three-state molecular orientation by suppressing simultaneous multiphoton excitation processes. This work provides an essential reference to quantum control of the populations and phases of rotational states, leading to the maximum degree of orientation within a three-state model.
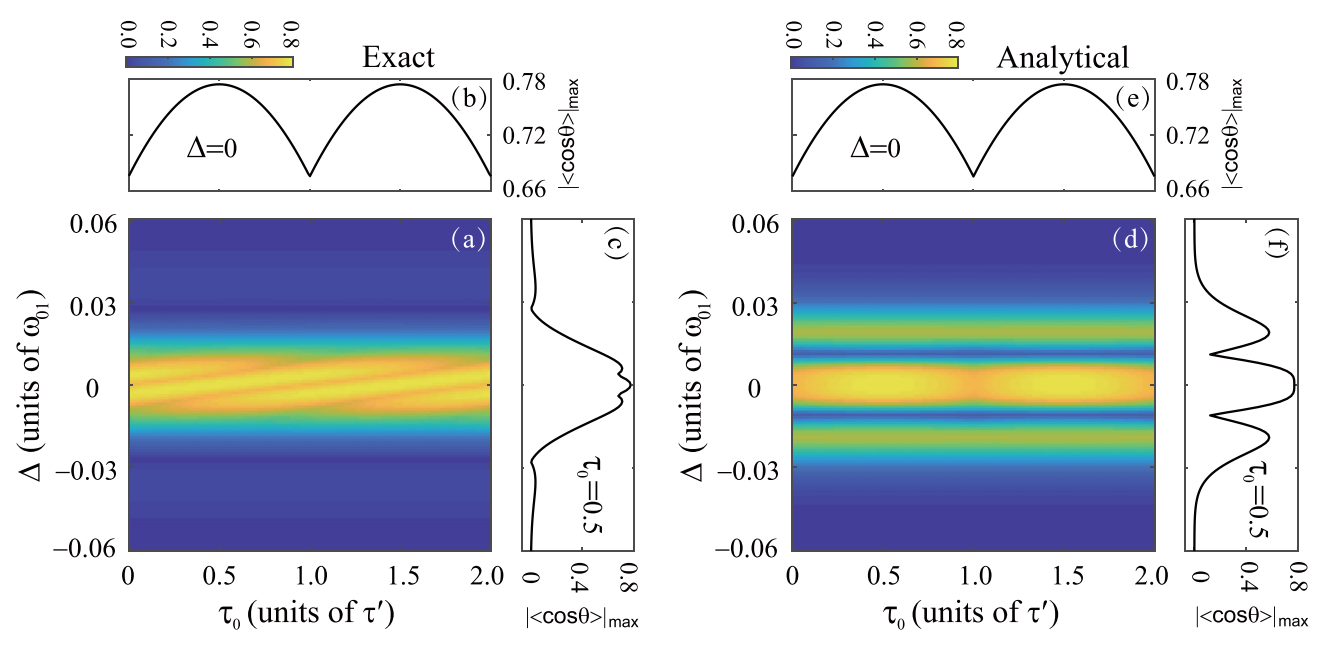

FIG. 10. (a)-(f) The same simulations as in Fig. 8, but for the second condition [Eq. (17)]. 
For practical applications, a compromise between maximal orientation efficiency and associated duration may be relevant. The present analytical model consisting of the lowest rotational states in a lower-dimensional subspace is beneficial for obtaining a long duration of the orientation. As shown in Fig. 1, the relative duration of orientation over which it remains above a given threshold of 0.5 is about $0.184 \tau$. By extending the model to a higher-dimensional subspace, additional rotational levels could significantly improve the maximal orientation. As explained in Ref. [44], the upper limit values of $\langle\cos \theta\rangle$ for $J_{\max }=3,4$, and 5 are $0.861,0.906$, and 0.932 , respectively. This opens a fundamental interesting and challenging question of whether an analytical model could be derived to include more rotational states.

In addition, the efficiency of orientation is subject to a drastic decrease with temperature, which is characterized by an additional average over all possible rotational states weighted by the Boltzmann distribution $[24,29,50]$. Our analysis that was based on the initial rotational state $|00\rangle$ can be extended to molecules initially in other rotational states. Then, the analytical model is able to take into account the thermal average. As an example, we can calculate that the maximum degree of orientation for molecules initially in a pure rotational state $|10\rangle$ and $|1 \pm 1\rangle$ is 0.7746 and 0.4472 , respectively. By considering the thermal average at a low temperature $T=2 \mathrm{~K}$, the maximum degree of orientation for the mixed-state molecules within the three-state model is decreased to 0.7507 . It remains a long-standing challenge to obtain high efficiency of orientation for the molecules beyond ultracold temperature.

\section{ACKNOWLEDGMENTS}

This work was supported by the National Natural Science Foundations of China (NSFC) under Grant No. 61973317.
[1] H. Stapelfeldt and T. Seideman, Colloquium: Aligning molecules with strong laser pulses, Rev. Mod. Phys. 75, 543 (2003).

[2] Y. Ohshima and H. Hasegawa, Coherent rotational excitation by intense nonresonant laser fields, Int. Rev. Phys. Chem. 29, 619 (2010).

[3] S. Fleischer, Y. Khodorkovsky, E. Gershnabel, Y. Prior, and I. Sh. Averbukh, Molecular alignment induced by ultrashort laser pulses and its impact on molecular motion, Isr. J. Chem. 52, 414 (2012).

[4] C. P. Koch, M. Lemeshko, and D. Sugny, Quantum control of molecular rotation, Rev. Mod. Phys. 91, 035005 (2019).

[5] J. J. Qiang, I. Tutunnikov, P. F. Lu, K. Lin, W. B. Zhang, F. H. Sun, Y. Silberberg, Y. Prior, I. S. Averbukh, and J. Wu, Echo in single vibrationally excited molecule, Nat. Phys. 16, 328 (2020).

[6] A. Goban, S. Minemoto, and H. Sakai, Laser-Field-Free Molecular Orientation, Phys. Rev. Lett. 101, 013001 (2008).

[7] O. Ghafur, A. Rouzée, A. Gijsbertsen, W. K. Siu, S. Stolte, and M. J. J. Vrakking, Impulsive orientation and alignment of quantum-state-selected NO molecules, Nat. Phys. 5, 289 (2009).

[8] S. De, I. Znakovskaya, D. Ray, F. Anis, N. G. Johnson, I. A. Bocharova, M. Magrakvelidze, B. D. Esry, C. L. Cocke, I. V. Litvinyuk, and M. F. Kling, Field-Free Orientation of CO Molecules by Femtosecond Two-Color Laser Fields, Phys. Rev. Lett. 103, 153002 (2009).

[9] I. Znakovskaya, M. Spanner, S. De, H. Li, D. Ray, P. Corkum, I. V. Litvinyuk, C. L. Cocke, and M. F. Kling, Transition between Mechanisms of Laser-Induced Field-Free Molecular Orientation, Phys. Rev. Lett. 112, 113005 (2014).

[10] K. Li, I. Tutunnikov, J. J. Qiang, J. Y. Ma, Q. Y. Song, Q. Y. Ji, W. B. Zhang, H. X. Li, F. H. Sun, X. C. Gong, H. Li, P. F. Lu, H. P. Zeng, Y. Prior, I. S. Averbukh, and J. Wu, All-optical field-free three-dimensional orientation of asymmetric-top molecules, Nat. Commun. 9, 5134 (2018).

[11] Y. Sugawara, A. Goban, S. Minemoto, and H. Sakai, Laserfield-free molecular orientation with combined electrostatic and rapidly-turned-off laser fields, Phys. Rev. A 77, 031403(R) (2008).
[12] I. Tutunnikov, J. Floß, E. Gershnabel, P. Brumer, I. Sh. Averbukh, A. A. Milner, and V. Milner, Observation of persistent orientation of chiral molecules by a laser field with twisted polarization, Phys. Rev. A 101, 021403(R) (2020).

[13] K. Brandt, M. E. Chiu, D. J. Watson, M. S. Tikhov, and R. M. Lambert, Chemoselective catalytic hydrogenation of acrolein on $\operatorname{Ag}(111)$ : Effect of molecular orientation on reaction selectivity, J. Am. Chem. Soc. 131, 17286 (2009).

[14] J. Wu, L. Ph. H. Schmidt, M. Kunitski, M. Meckel, S. Voss, H. Sann, H. Kim, T. Jahnke, A. Czasch, and R. Dörner, Multiorbital Tunneling Ionization of the CO Molecule, Phys. Rev. Lett. 108, 183001 (2012).

[15] Y.-P. Chang, K. Długołecki, J. Küpper, D. Rösch, D. Wild, and S. Willitsch, Specific chemical reactivities of spatially separated 3-aminophenol conformers with cold $\mathrm{Ca}^{+}$ions, Science 342, 98 (2013).

[16] P. Rotter, B. A. J. Lechner, A. Morherr, D. M. Chisnall, D. J. Ward, A. P. Jardine, J. Ellis, W. Allison, B. Eckhardt, and G. Witte, Coupling between diffusion and orientation of pentacene molecules on an organic surface, Nat. Mater. 15, 397 (2016).

[17] C.-C. Shu, Y. Guo, K.-J. Yuan, D. Y. Dong, and A. D. Bandrauk, Attosecond all-optical control and visualization of quantum interference between degenerate magnetic states by circularly polarized pulses, Opt. Lett. 45, 960 (2020).

[18] J. P. Li, Q. B. Zhang, L. Li, X. S. Zhu, T. F. Huang, P. F. Lan, and P. X. Lu, Orientation dependence of high-order harmonic generation in nanowire, Phys. Rev. A 99, 033421 (2019).

[19] G. Karras, E. Hertz, F. Billard, B. Lavorel, J.-M. Hartmann, O. Faucher, E. Gershnabel, Y. Prior, and I. S. Averbukh, Orientation and Alignment Echoes, Phys. Rev. Lett. 114, 153601 (2015).

[20] H. Zhang, B. Lavorel, F. Billard, J.-M. Hartmann, E. Hertz, O. Faucher, J. Y. Ma, J. Wu, E. Gershnabel, Y. Prior, and I. S. Averbukh, Rotational Echoes as a Tool for Investigating Ultrafast Collisional Dynamics of Molecules, Phys. Rev. Lett. 122, 193401 (2019).

[21] S. Fleischer, Y. Zhou, R. W. Field, and K. A. Nelson, Molecular Orientation and Alignment by Intense Single-Cycle $\mathrm{THz}$ Pulses, Phys. Rev. Lett. 107, 163603 (2011). 
[22] S. Fleischer, R. W. Field, and K. A. Nelson, Commensurate Two-Quantum Coherences Induced by Time-Delayed THz Fields, Phys. Rev. Lett. 109, 123603 (2012).

[23] N. E. Henriksen, Molecular alignment and orientation in short pulse laser fields, Chem. Phys. Lett. 312, 196 (1999).

[24] M. Machholm and N. E. Henriksen, Field-Free Orientation of Molecules, Phys. Rev. Lett. 87, 193001 (2001).

[25] C. M. Dion, A. Keller, and O. Atabek, Orienting molecules using half-cycle pulses, Eur. Phys. J. D 14, 249 (2001).

[26] D. Sugny, A. Keller, O. Atabek, D. Daems, S. Guérin, and H. R. Jauslin, Time-dependent unitary perturbation theory for intense laser-driven molecular orientation, Phys. Rev. A 69, 043407 (2004).

[27] D. Daems, S. Guérin, D. Sugny, and H. R. Jauslin, Efficient and Long-Lived Field-Free Orientation of Molecules by a Single Hybrid Short Pulse, Phys. Rev. Lett. 94, 153003 (2005).

[28] E. Gershnabel, I. Sh. Averbukh, and R. J. Gordon, Orientation of molecules via laser-induced anti-alignment, Phys. Rev. A 73, 061401(R) (2006).

[29] C.-C. Shu, K.-J. Yuan, W.-H. Hu, J. Yang, and S.-L. Cong, Controlling the orientation of polar molecules in a rovibrationally selective manner with an infrared laser pulse and a delayed half-cycle pulse, Phys. Rev. A 78, 055401 (2008).

[30] C.-C. Shu, K.-J. Yuan, W.-H. Hu, and S.-L. Cong, Carrierenvelope phase-dependent field-free molecular orientation, Phys. Rev. A 80, 011401(R) (2009).

[31] C.-C. Shu, K.-J. Yuan, W.-H. Hu, and S.-L. Cong, Field-free molecular orientation with terahertz few-cycle pulses, J. Chem. Phys. 132, 244311 (2010).

[32] K. Kitano, N. Ishii, and J. Itatani, High degree of molecular orientation by a combination of $\mathrm{THz}$ and femtosecond laser pulses, Phys. Rev. A 84, 053408 (2011).

[33] C.-C. Shu and N. E. Henriksen, Field-free molecular orientation induced by single-cycle THz pulses: The role of resonance and quantum interference, Phys. Rev. A 87, 013408 (2013).

[34] K. N. Egodapitiya, L. Sha, and R. R. Jones, Terahertz-Induced Field-Free Orientation of Rotationally Excited Molecules, Phys. Rev. Lett. 112, 103002 (2014).

[35] R. Damari, S. Kallush, and S. Fleischer, Rotational Control of Asymmetric Molecules: Dipole-Versus Polarizability-Driven Rotational Dynamics, Phys. Rev. Lett. 117, 103001 (2016).

[36] J. Ortigoso, Mechanism of molecular orientation by singlecycle pulses, J. Chem. Phys. 137, 044303 (2012).
[37] M. Lapert and D. Sugny, Field-free molecular orientation by terahertz laser pulses at high temperature, Phys. Rev. A 85, 063418 (2012).

[38] D. Sugny, S. Vranckx, M. Ndong, N. Vaeck, O. Atabek, and M. Desouter-Lecomte, Control of molecular dynamics with zero-area fields: Application to molecular orientation and photofragmentation, Phys. Rev. A 90, 053404 (2014).

[39] L. H. Coudert, Optimal orientation of an asymmetric top molecule with terahertz pulses, J. Chem. Phys. 146, 024303 (2012).

[40] R. Tehini, K. Hamraoui, and D. Sugny, Shaping of the time evolution of field-free molecular orientation by $\mathrm{THz}$ laser pulses, Phys. Rev. A 99, 033419 (2019).

[41] L. Xu, I. Tutunnikov, E. Gershnabel, Y. Prior, and I. S. Averbukh, Long-Lasting Molecular Orientation Induced by a Single Terahertz Pulse, Phys. Rev. Lett. 125, 013201 (2020).

[42] C.-C. Shu, Q.-Q. Hong, Y. Guo, and N. E. Henriksen, Orientational quantum revivals induced by a single-cycle terahertz pulse, Phys. Rev. A 102, 063124 (2020).

[43] D. Sugny, A. Keller, O. Atabek, D. Daems, C. M. Dion, S. Gurin, and H. R. Jauslin, Reaching optimally oriented molecular states by laser kicks, Phys. Rev. A 69, 033402 (2004).

[44] S. Wang and N. E. Henriksen, Optimal field-free molecular orientation with nonresonant two-color adiabatic-turn-on and sudden-turn-off laser pulses, Phys. Rev. A 102, 063120 (2020).

[45] G. Shchedrin, C. O’Brien, Y. Rostovtsev, and M. O. Scully, Analytic solution and pulse area theorem for three-level atoms, Phys. Rev. A 92, 063815 (2015).

[46] Y. Guo, X. B. Luo, S. Ma, and C.-C. Shu, All-optical generation of quantum entangled states with strictly constrained ultrafast laser pulses, Phys. Rev. A 100, 023409 (2019).

[47] Y. Guo, C.-C. Shu, D. Dong, and F. Nori, Vanishing and Revival of Resonance Raman Scattering, Phys. Rev. Lett. 123, 223202 (2019).

[48] S. Blanes, F. Casas, J. A. Oteo, and J. Ros, The Magnus expansion and some of its applications, Phys. Rep. 470, 151 (2009).

[49] J. Salomon, C. M. Dion, and G. Turinici, Optimal molecular alignment and orientation through rotational ladder climbing, J. Chem. Phys. 123, 144310 (2005).

[50] D. Sugny, A. Keller, O. Atabek, D. Daems, C. M. Dion, S. Guérin, and H. R. Jauslin, Control of mixed-state quantum systems by a train of short pulses, Phys. Rev. A 72, 032704 (2005). 